\title{
Ultrastructural quantification of collagen in human chordae tendineae
}

\author{
B K B Berkovitz, C Rollinson
}

\begin{abstract}
Objective-To determine collagen fibril diameter distributions in the chordae tendineae and to see whether there are any differences between right and left sides of the human heart.

Design-Collagen fibril diameters and the percentage volume occupied by collagen fibrils (as opposed to ground substance) were determined by means of a planimeter with a digitising tablet from electron micrographs printed at a magnification of 136; 000 .

Material-Human chordae tendineae were obtained at postmortem examination from seven subjects aged 50-75 years.
\end{abstract}

Measurements and main resultsHistograms of collagen fibril diameter distributions showed a sharp, unimodal distribution. The mean collagen fibril diameters associated with the tricuspid and mitral valves were $41 \cdot 1 \mathrm{~nm}$ and 40.5 nm respectively. The percentage volume occupied by collagen was about $39 \%$ for each valve. There were no significant differences for any of these values between the right and left sides of the heart.

Conclusions-The greater pressures present on the left side of the heart are not reflected in any difference in collagen fibril diameters within the chordae tendineae compared with the right side.

\section{(Br Heart ₹ 1993;69:246-249)}

Pathological changes in the myocardium are associated with changes in the connective tissue. The overall amounts of collagen increases in ventricular hypertrophy. ${ }^{1-3}$ This is accompanied by an increase in the number and diameter of collagen fibres and a considerable distortion of the weave. ${ }^{4}$ The increase in collagen content implies that the connective tissue component of the heart can be remodelled. ${ }^{13}$ The percentage of collagen turning over in the normal heart is reported as being between less than $1 \%$ and up to $6 \% .^{256}$ Turnover is greater in the left ventricle than in the right and is increased with hypertrophy. ${ }^{2}$ The reported diameter of individual collagen fibrils in the myocardium is 30-70 nm.

Data on collagen fibrils in the chordae tendineae of the right and left human atrioventricular valves (means are for 6 micrographs)

\begin{tabular}{|c|c|c|}
\hline Specimen & Tricuspid & Mitral \\
\hline $\begin{array}{l}\text { Case A (female aged } 75) \text { : } \\
\text { Overall mean (1SD) fibril diameter (nm) } \\
\text { Mean SD for each micrograph of the group } \\
\text { Mean (1SD) volume of extracellular matrix occupied by collagen }(\%)\end{array}$ & $\begin{array}{l}40 \cdot 5(4 \cdot 3) \\
(6 \cdot 9) \\
38 \cdot 9(2 \cdot 1)\end{array}$ & $\begin{array}{l}39 \cdot 1(3 \cdot 5) \\
(6 \cdot 5) \\
34 \cdot 5(2 \cdot 3)\end{array}$ \\
\hline $\begin{array}{l}\text { Case B (female aged } 61) \text { : } \\
\text { Overall mean (1SD) fibril diameter (nm) } \\
\text { Mean SD for each micrograph of the group } \\
\text { Mean (1SD) volume of extracellular matrix occupied by collagen }(\%)\end{array}$ & $\begin{array}{l}46 \cdot 0(5 \cdot 3) \\
(7 \cdot 6) \\
38 \cdot 0(5 \cdot 2)\end{array}$ & $\begin{array}{l}42 \cdot 2(6 \cdot 0) \\
(7 \cdot 0) \\
33 \cdot 3(5 \cdot 8)\end{array}$ \\
\hline $\begin{array}{l}\text { Case C (male aged 50): } \\
\text { Overall mean (1SD) fibril diameter (nm) } \\
\text { Mean SD for each micrograph of the group } \\
\text { Mean (1SD) volume of extracellular matrix occupied by collagen (\%) }\end{array}$ & $\begin{array}{l}40 \cdot 1(4 \cdot 5) \\
(6 \cdot 6) \\
42 \cdot 2(3 \cdot 2)\end{array}$ & $\begin{array}{l}45 \cdot 2(4 \cdot 3) \\
(6 \cdot 4) \\
39 \cdot 4(2 \cdot 4)\end{array}$ \\
\hline $\begin{array}{l}\text { Case } \mathrm{D} \text { (female aged } 67) \text { : } \\
\text { Overall mean (1SD) fibril diameter (nm) } \\
\text { Mean SD for each micrograph of the group } \\
\text { Mean (ISD) volume of extracellular matrix occupied by collagen (\%) }\end{array}$ & $\begin{array}{l}37 \cdot 7(2 \cdot 0) \\
(6 \cdot 3) \\
33 \cdot 6(8 \cdot 1)\end{array}$ & $\begin{array}{l}36 \cdot 1(2 \cdot 2) \\
(6 \cdot 5) \\
42 \cdot 7(5 \cdot 2)\end{array}$ \\
\hline $\begin{array}{l}\text { Case É: } \\
\text { Overall mean (1SD) fibril diameter (nm) } \\
\text { Mean SD for each micrograph of the group } \\
\text { Mean (ISD) volume of extracellular matrix occupied by collagen (\%) }\end{array}$ & $\begin{array}{l}41 \cdot 0(6 \cdot 6) \\
(6 \cdot 8) \\
38 \cdot 8(6 \cdot 6)\end{array}$ & $\begin{array}{l}42 \cdot 9(5 \cdot 4) \\
(9 \cdot 5) \\
40 \cdot 6(4 \cdot 6)\end{array}$ \\
\hline $\begin{array}{l}\text { Case F: } \\
\text { Overall mean (1SD) fibril diameter (nm) } \\
\text { Mean SD for each micrograph of the group } \\
\text { Mean (ISD) volume of extracellular matrix occupied by collagen (\%) }\end{array}$ & $\begin{array}{l}43 \cdot 7(5 \cdot 6) \\
(5 \cdot 8) \\
48 \cdot 6(5 \cdot 4)\end{array}$ & $\begin{array}{l}41.8(3.7) \\
(6.0) \\
43.4(3.2)\end{array}$ \\
\hline $\begin{array}{l}\text { Case G: } \\
\text { Overall mean (1SD) fibril diameter (nm) } \\
\text { Mean SD for each micrograph of the group } \\
\text { Mean (1SD) volume of extracellular matrix occupied by collagen (\%) }\end{array}$ & $\begin{array}{l}38 \cdot 7(7 \cdot 6) \\
(5 \cdot 4) \\
32 \cdot 2(8 \cdot 5)\end{array}$ & $\begin{array}{l}36 \cdot 5(2 \cdot 0) \\
(6 \cdot 1) \\
35 \cdot 1(3 \cdot 8)\end{array}$ \\
\hline $\begin{array}{l}\text { Mean: } \\
\text { Overall mean (1SD) fibril diameter (nm) } \\
\text { Mean (1SD) volume of extracellular matrix occupied by collagen (\%) }\end{array}$ & $\begin{array}{l}41.1(2.9) \\
38.9(5.5)\end{array}$ & $\begin{array}{l}40 \cdot 5(3 \cdot 4) \\
38 \cdot 4(4 \cdot 1)\end{array}$ \\
\hline
\end{tabular}

Biomedical Scienc :s

Division, King's College London B K B Berkovitz C Rollinson

Correspondence to Dr B K B Berkovitz Anatomy and Human Biology Group, Biomedical Science Division, King's College London, Strand, London WC2R 2LS.

Accepted for publication 21 September 1992 
Figure 1 Electron micrograph of fibroblast from a chorda tendineae showing some preservation of the intracellular organelles.

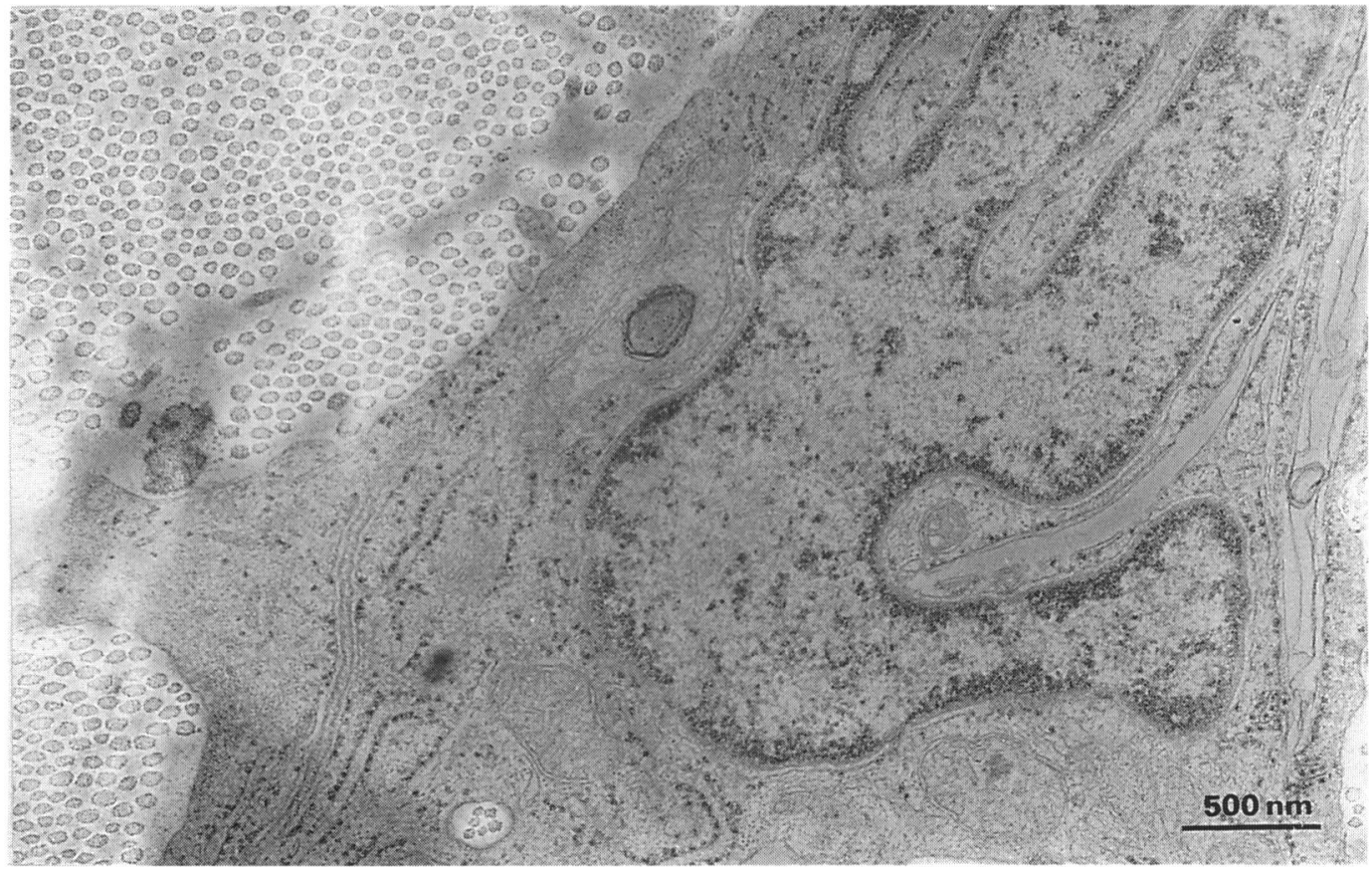

The studies described above that indicate the importance of collagen in the functioning of the heart have primarily been of the myocardium. The chordae tendineae are affected in many heart disorders, and though their gross anatomy has been well described ${ }^{89}$ there is little detailed information about the chordae tendineae in disease. Fragmentation of collagen ${ }^{10}{ }^{11}$ and accumulation of myxomatous material was reported in association with a floppy valve but there was no abnormal periodicity in the collagen fibrils. ${ }^{10}$ Caulfield and Kaslon reported that in conditions associated with spontaneous rupture of the chordae tendineae there are multiple and diffuse areas of collagen degeneration. ${ }^{12}$ The results from another study do not support the view that collagen degeneration occurs and it has been concluded that rupture is the consequence of abnormal mechanical strain. ${ }^{13}$

To understand the changes occurring in the chordae tendineae in disease, requires a knowledge of their detailed structure in the normal state. In addition, because the right and left ventricles of the heart generate different pressures, the chordae tendineae provide a useful experimental model for correlating structure and function in connective tissues. In sheep and rabbit heart, there is evidence that collagen fibril diameters are greater in the chordae tendineae of the mitral valve than those of the tricuspid valve. ${ }^{14}$ Because collagen fibril dimensions may reflect the biomechanical properties to which the connective tissue is subjected, ${ }^{15} 16$ the aim of the present study was to obtain baseline data on collagen fibril diameter distributions in human chordae tendineae.

\section{Patients and methods}

Chordae tendineae were dissected from the right and left sides of the hearts of seven human subjects (aged 50-75) at postmortem examination. The precise age of four of the subjects was known (table). The full medical history of each subject was not known, but in each case the immediate cause of death was unrelated to specific chronic heart conditions such as hypertension. The chordae tendineae were fixed about 10 hours after death in $2.5 \%$ gluteraldehyde in $0.1 \mathrm{M}$ phosphate buffer, postfixed in $1 \%$ osmium tetroxide in phosphate buffer, dehydrated through a graded series of ethanols and propylene oxide, and embedded in Epon 812. Ultrathin sections of the chordae tendineae were cut in the transverse plane of the chordae near the base, stained with uranyl acetate and lead citrate, and viewed in an electron microscope.

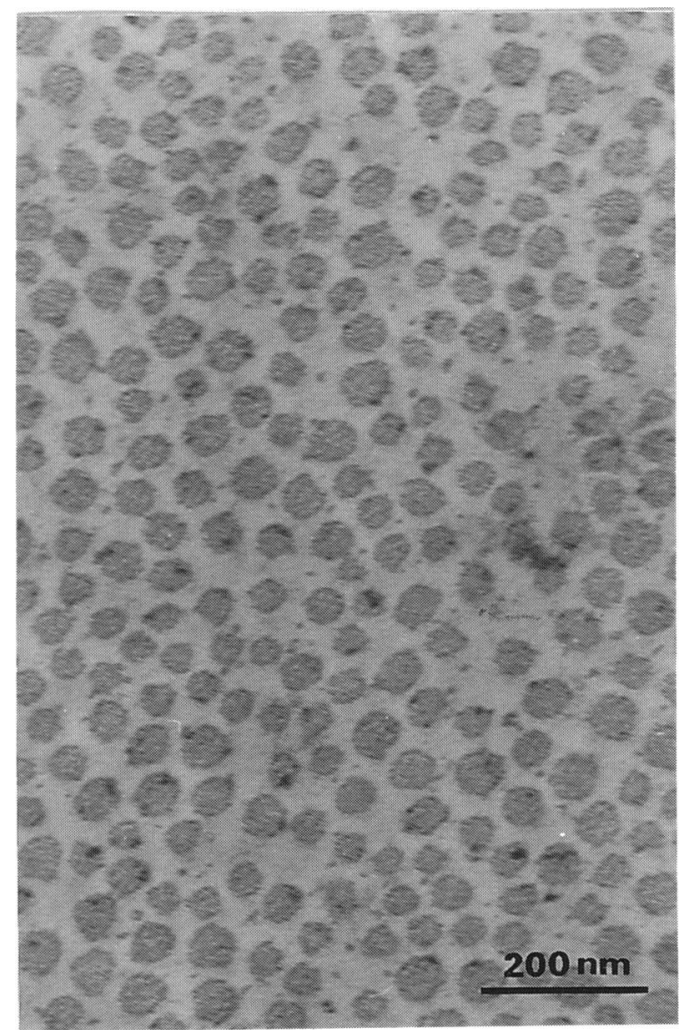

Figure 2 Electron micrograph showing collagen fibrils cut transversely in a chorda tendineae. 
Figure 3 Histogram of collagen fibril diameter distributions in seven specimens (see table).
Six micrographs for each of the tricuspid and mitral valves were obtained from the chordae of each subject, and these were printed at a final magnification of $\times 136000$. To ensure consistent magnification, a cross-grating replica was used for calibration. The minimum diameters of collagen fibrils and
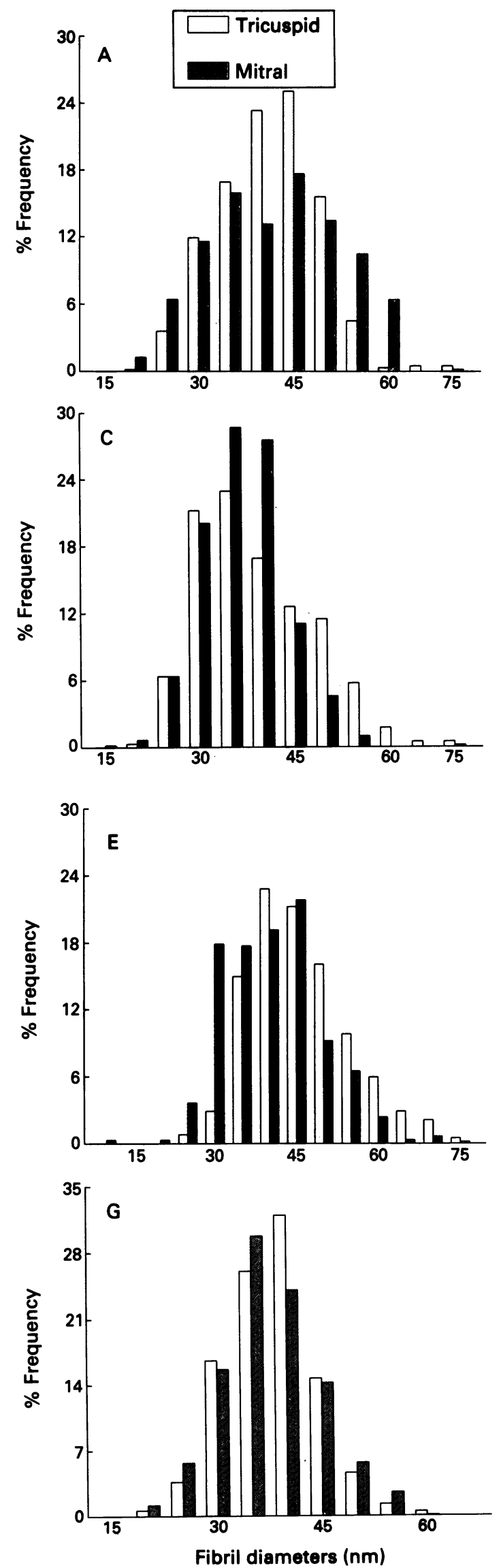

also the percentage volume of a fibre bundle occupied by collagen were measured by means of a planimeter with a digitising tablet (Digiplan, Kontron, Munich). We started from one corner of each micrograph and measured the diameter of each collagen fibril, $\underline{\underline{T}}$ radiating out in an arc until at least 100 fibrils $I$
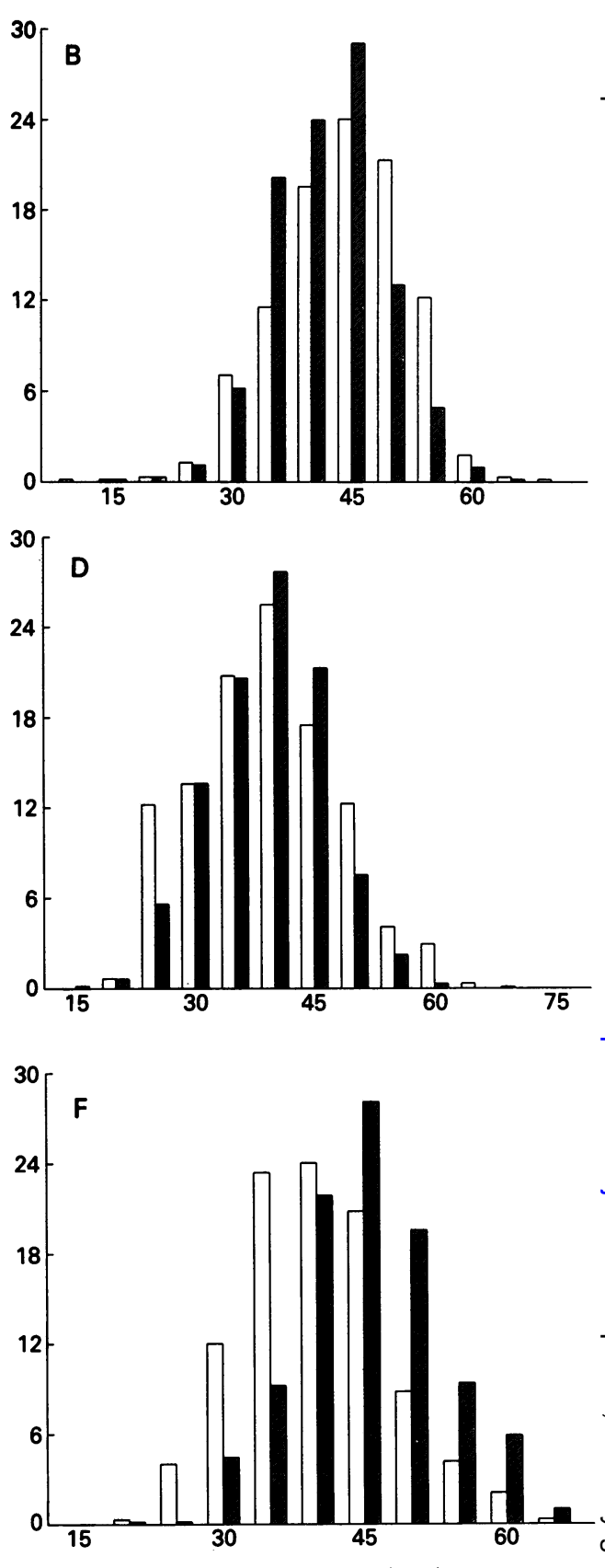

Fibril diameters $(\mathrm{nm})$ 
Figure 4 Histogram of data from all seven specimens. $(n=4361$ for tricuspid valves, $n=4371$ for mitral valves).

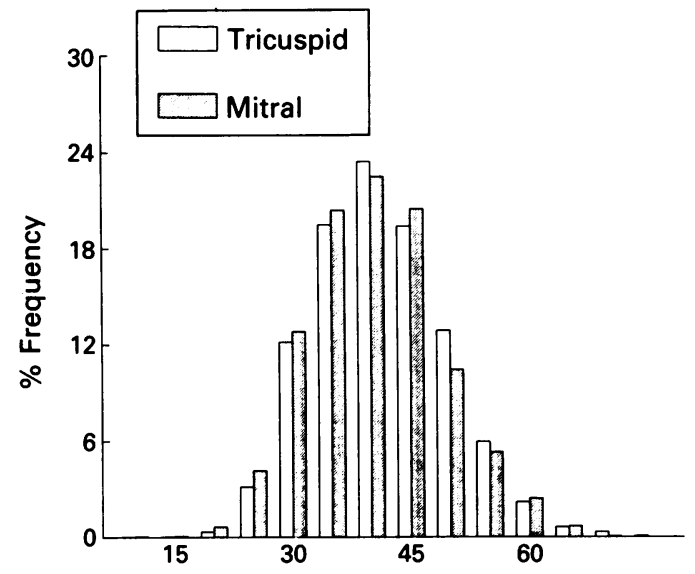

Fibril diameters $(\mathrm{nm})$

had been studied. More than 4350 fibrils were measured for both the tricuspid and mitral valves. The total volume occupied by the collagen fibrils in a circumscribed area (diameter $370 \mathrm{~nm}$ ) containing about 30 fibrils was measured and expressed as a percentage (the remainder of the area was occupied by the ground substance). The significance of any differences between the data was tested by an analysis of variance.

\section{Results}

The preservation of some of the intracellular organelles within the fibroblasts suggested that there was fairly good preservation of the chordae tendineae (fig 1). The table shows the collagen fibril diameters in the seven subjects. Collagen fibril diameters were small (mean approximately $40 \mathrm{~nm}$ ) and unimodally distributed (fig 2). Figure 3 shows the individual histograms for the seven subjects and fig 4 shows the histogram for the combined data. No significant difference could be discerned between mean collagen fibril diameters from the right and left sides of the heart.

Collagen fibrils occupied on average about $40 \%$ of the volume of the fibre bundles: the remaining $60 \%$ was occupied by ground substance. The values for the right and left sides of the heart were not significantly different.

\section{Discussion}

Though the material we studied was not fixed for some hours after death, the appearance of the intracellular organelles associated with the fibroblasts suggested good preservation. The present findings indicate that the mean diameter of collagen fibrils within the chordae tendineae of the human heart is about $40 \mathrm{~nm}$ and that size is unimodally distributed. Certain tendons show a bimodal distribution with many large fibrils with a maximum diameter of almost $400 \mathrm{~nm} .^{1516}$ These findings suggest that collagen fibril diameters may be determined by not only the absolute size of the load on the tendon but also by whether the load is continuous or intermittent.

No significant difference was observed between collagen fibril diameters of the chordae tendineae from the right and left sides of the human heart, despite the obvious differ- ences in blood pressure between the two ventricles of the heart. This differs from results obtained for a similar type of study in the sheep and rabbit heart. No obvious explanation can yet be offered to account for this difference. However, three additional studies could be undertaken to provide further data on the possible relation between collagen fibril diameters and function. First, it would be appropriate to carry out a similar investigation on a much younger age group. Second, analysis of fibril diameters from neonates may also provide useful data because the right ventricle would have been mainly bypassed up to this period. Third, analysis of the chordae tendineae from individuals with chronic hypertension would indicate whether collagen fibril diameters can increase with increased blood pressure. There is a mutant strain of rat (SHR strain) in which hypertension develops spontaneously. Changes in collagen of the chordae tendineae during hypertension are being studied in this animal model.

In the chordae tendineae collagen fibrils occupy about $40 \%$ of the extracellular matrix and there is no difference between valves from the right and left sides of the heart. These values more closely resemble those associated with connective tissues under compression rather than tension, ${ }^{15}$ and they again indicate the importance of the nature of the load (that is, intermittent or continuous) in regulating fibril diameters.

We thank the mortuary staff at King's College Hospital for providing the material.

1 Pearlman ES, Weber KT, Janicki JS et al. Muscle fibre orientation and connective tissue content in hypertrophied human heart. Lab Invest 1986;46:158-64.

2 Turner JE, Oliver, MH, Guerrard D, Laurent GJ. Collagen metabolism during right ventricular hypertrophy following induced lung injury. $A m \mathcal{F}$ Physiol 1986;251:H915-9.

3 Weber KT, Janicki JS, Shroff SG, et al. Collagen remodelling of the pressure overload, hypertrophied non-human primate myocardium. Giri Res 1988;67:757-65.

4 Abrahams C, Janicki S, Weber KT. Myocardial hypertrophy in Macaca fascicularis. Lab Invest 1987;56:676-83.

5 Laurent GJ, Sparrow MP, Bates PC, Millward DJ. Collagen content and turnover in cardiac and skeletal muscles of the adult fowl and the changes during stretch-induced growth. Biochem $₹$ 1978;176:419-27.

6 Bonnin CM, Sparrow MP, Taylor RR. Collagen synthesis and content in right ventricular hypertrophy in the dog. Am $\mathcal{f}$ Physiol;10:H703-13.

7 Robinson TF, Cohen-Gould L, Factor SM. Skeletal framework of mammalian heart muscle. Lab Invest 1983;49:482-98.

8 Lam JH, Ranganathan N, Wigle ED et al. Morphology of the human mitral valve. 1 . Chordae tendineae Circulation 1970;41:449-67.

9 Roberts WC, Cohen LS. Left ventricular papillary muscles. Circulation 1972;46:138-54.

10 Davies MJ, Moore BP, Braimbridge MV. The floppy mitral valve. Br Heart $\mathscr{f}$ 1978;40:468-81.
mies

11 Baker PB, Bansal G, Boudoulas H et al. Floppy mitral valve chordae tendineae. Hum Pathol 1988;19:507-12.

12 Caulfield JB, Kastor JA. Connective tissue abnormalities in spontaneous rupture of chordae tendineae. Arch Pathol Lab Med 1971;91:537-41.

13 Scott-Jupp W, Barnett NL, Gallagher PJ, et al. Ultrastructural changes in spontaneous rupture of mitral chordae tendineae. F Pathol 1981;133:185-201.

14 Berkovitz BK, Robinson S. Ultrastructural quantification of collagen fibrils in the chordae tendineae of the sheep and rabbit. 7 Anat 1991;178:127-32.

15 Merrilees MJ, Flint MH. Ultrastructural study of tension and pressure zones in a rabbit flexor tendon. Am $\mathcal{F}$ Anat and pressure zones

16 Parry DA, Barnes GR, Craig AS. A comparison of the size distribution of collagen fibrils in connective tissues as a function of age and a possible relation between fibril size distribution and mechanical properties. Proc $R$ Soc Lond [Biol] 1981;203:305-21. 